University of Texas at El Paso

ScholarWorks@UTEP

$12-2009$

\title{
Symmetries: A General Approach to Integrated Uncertainty Management
}

Vladik Kreinovich

The University of Texas at El Paso, vladik@utep.edu

Hung T. Nguyen

Songsak Sriboonchitta

Follow this and additional works at: https://scholarworks.utep.edu/cs_techrep

Part of the Computer Engineering Commons

Comments:

Technical Report: UTEP-CS-09-31a

Published in: Van-Nam Huynh et al. (eds.), Integrated Uncertainty Management and

Applications, Springer-Verlag, Berlin, Heidelberg, 2010, Vol. AISC 68, pp. 141-152.

\section{Recommended Citation}

Kreinovich, Vladik; Nguyen, Hung T.; and Sriboonchitta, Songsak, "Symmetries: A General Approach to Integrated Uncertainty Management" (2009). Departmental Technical Reports (CS). 59.

https://scholarworks.utep.edu/cs_techrep/59

This Article is brought to you for free and open access by the Computer Science at ScholarWorks@UTEP. It has been accepted for inclusion in Departmental Technical Reports (CS) by an authorized administrator of ScholarWorks@UTEP.For more information, please contact Iweber@utep.edu. 


\title{
Symmetries: A General Approach to Integrated Uncertainty Management
}

\author{
Vladik Kreinovich, Hung T. Nguyen, and Songsak Sriboonchitta
}

\begin{abstract}
We propose to use symmetries as a general approach to maintaining different types of uncertainty, and we show how the symmetry approach can help, especially in economics-related applications.
\end{abstract}

\section{Why Symmetries}

Formulation of the problem. Our knowledge is rarely complete, we rarely have absolutely certainty. Uncertainty is present in different areas of knowledge. As a result, in many different areas of knowledge, different techniques and approaches have been developed to describe and process uncertainty. For example, in logical-type descriptions of knowledge typical for expert systems and Artificial Intelligence, formalisms like probabilistic logic and fuzzy logic have been developed to process uncertainty. In engineering-oriented probability-type descriptions, probability-related approaches have been developed such as the Dempster-Shafer approach, imprecise probabilities approach, etc.

To solve complex real-life problems, we must takes into account knowledge form different areas. Since these different pieces of knowledge come with uncertainty, we must therefore jointly manage different types of uncertainty. We therefore need a general approach that would take care of different types of uncertainty.

Vladik Kreinovich

University of Texas at El Paso, El Paso, TX 79968, USA, e-mail: vladik@utep.edu and

Faculty of Economics, Chiang Mai University, Chiang Mai 50200, Thailand

Hung T. Nguyen

New Mexico State University, Las Cruces NM 88003, USA, e-mail: hunguyen@nmsu.edu and and Faculty of Economics, Chiang Mai University, Chiang Mai 50200, Thailand

Songsak Sriboonchitta

Faculty of Economics, Chiang Mai University, Chiang Mai 50200, Thailand, e-mail: songsak@econ.cmu.ac.th 
Symmetry: a fundamental property of the physical world. The reason why we are gaining and processing knowledge is that we want to predict the processes of the physical world, predict the results of different possible actions - and thus, select the action whose results are most beneficial.

On the fundamental level, the very possibility to predict the processes and the results of different actions comes from the fact that we have observed similar situations, we remember the outcomes of these similar situations, and we expect that the outcomes will be similar.

For example, if in the past, we dropped a ball several times and every time, it fell down, then in a new situation we expect the ball to fall down as well. In the past, we may have been at different locations, at different moments of time, oriented differently, but the results were the same. Thus, we conclude that the outcome of this simple drop-the-ball experiment will be the same.

In mathematical terms, the similarity between different situations corresponds to symmetry, and the fact that the result is the same for similar situations is usually described as invariance.

In these terms, we can say, e.g., that the results of the "drop-the-ball" gravitational experiment are invariant relative to shifting the location, rotating (= changing orientation), and shifting in time.

The notion of symmetry is not only methodologically fundamental: symmetries are one of the main tools of modern physics; see, e.g., [4].

Because of the fundamental nature of symmetries in describing the physical world, it is reasonable to try to use symmetries for describing uncertainty as well.

In this paper, we describe the basic symmetries, explain how they explain the basic uncertainty-related formulas, and show they symmetries also help in explaining and designing uncertainty-related algorithms - thus providing a reasonable foundation for integrated uncertainty measurement.

Basic symmetries: scaling and shift. In applied computations, we deal with the numerical values of a physical quantity. For most quantity, however, the numerical values depend not only on the quantity itself, but also on the unit in which we measure this quantity. For example, we can measure length in feet or in $\mathrm{cm}$.

Since the choice of a measuring unit is usually an arbitrary convention, it is reasonable to require that all the formulas remain invariant when we change these arbitrary units. How can we describe this invariance in precise terms? If we replace a measuring unit by another unit which is $\lambda$ times smaller, then the corresponding numerical values are multiplied by $\lambda: x \rightarrow \lambda \cdot x$. For example, when we replace a meter with a 100 times smaller unit $(\mathrm{cm})$, all numerical values are multiplied by 100: $1.7 \mathrm{~m}$ becomes $170 \mathrm{~cm}$. This transformation is called scaling.

For many units such as time (and temperature), there is another arbitrariness: in selecting the starting point. It is well known that in different calendars, the starting date is different. If we replace the original starting point with a new one which is $s$ units smaller, then the original numerical value increases by $s: x \rightarrow x+s$. This transformation is called shift.

Together, scaling and shifts form linear transformations $x \rightarrow a \cdot x+b$. 
Basic nonlinear symmetries. Sometimes, in addition to linear symmetries, a system also has nonlinear symmetries. In this case, the class of all possible symmetries contains all linear functions and some nonlinear functions as well.

If a system is invariant under transformations $f$ and $g$, then we can conclude that it is invariant also under their composition $f \circ g$, and also invariant under the inverse transformation $f^{-1}$. In mathematical terms, this means that symmetries form a group.

To describe a generic linear transformation, we need 2 parameters: $a$ and $b$. To describe a more general nonlinear transformation, we may need more parameters. In practice, at any given moment of time, we can only store and describe finitely many parameters. Thus, it is reasonable to restrict ourselves to finite-dimensional groups, i.e., groups all elements of which can be characterized by finitely many parameters. It is also reasonable to assume that the group is connected (i.e., there is a continuous transition between every two transformations) and that the dependence on the parameters is smooth (differentiable) - i.e., in mathematical terms, that we have a Lie group. How can we describe all finite-dimensional Lie groups of transformations of the set of real numbers $R$ onto itself that contain all linear transformations? Norbert Wiener asked [19] to classify such groups for an $n$-dimensional space with arbitrary $n$, and this classification was obtained in [16]. In our case (when $n=1$ ) the only possible groups are the group of all linear transformations and the group of all fractionally-linear transformations $x \rightarrow(a \cdot x+b) /(c \cdot x+d)$. In both cases the group consists only of fractionally linear transformations.

Symmetries explain the basic formulas of different uncertainty formalisms. Let us show that the above basic symmetries provide a unified basis for explaining many uncertainty-related heuristic formulas. These results are described, in detail, in [11].

Let us start with neural networks, in which the main heuristic (empirically justified) formula is the formula for the nonlinear activation function $f(x)=1 /\left(1+e^{-x}\right)$. As we have mentioned, a change in the starting point of a measuring scale replaces the numerical value $x$ with a new value $x+s$. It is reasonable to require that the new output $f(x+s)$ be equivalent to the original one $f(x)$ modulo an appropriate transformation. Since, as we mentioned, all appropriate transformations are fractionally linear, we thus conclude that $f(x+s)$ must be related to $f(x)$ by a fractionally linear transformation, i.e., that $f(x+s)=(a(s) \cdot f(x)+b(s)) /(c(s) \cdot f(x)+d(s))$ for some values $a(s), \ldots, d(s)$. Differentiating both sides by $s$ and equating $s$ to 0 , we get a differential equation for the unknown function $f(x)$ whose solution is the above activation function - which can thus be explained by symmetries.

If, instead of a shift, we consider scaling of $x$, we get a different activation function - which has also been successfully used in neural networks.

Similarly, symmetries can help explain heuristic formulas of fuzzy logic. Indeed, in fuzzy logic, the main quantity is the certainty (membership) degree $a$. One way to define the certainty degree $a$ of a statement $S$ is by polling $n$ experts and taking, as $a=m / n$, the fraction of those who believe $S$ to be true. To make this estimate more accurate, we can go beyond top experts and ask $n^{\prime}$ other experts as well. In the presence of top experts, however, other experts may either remain shyly silent or shyly confirm the majority's opinion. In the first case, the degree reduces from 
$a=m / n$ to $a^{\prime}=m /\left(n+n^{\prime}\right)$, i.e., to $a^{\prime}=\lambda \cdot a$, where $\lambda=n /\left(n+n^{\prime}\right)$. In the second case, $a$ changes to $a^{\prime}=\left(m+m^{\prime}\right) /\left(n+m^{\prime}\right)-$ a linear transformation. In general, we get all linear transformations.

We can describe the degree of certainty $d(S)$ in a statement $S$ by its own degree of certainty, or, alternatively, by a degree of certainty in, say, $S \& S_{0}$ for some statement $S_{0}$. It is reasonable to require that the corresponding transformation $d(S) \rightarrow d\left(S \& S_{0}\right)$ belong to the finite-dimensional transformation group that contains all linear transformations - thus, that it is fractionally linear. This requirement explains many empirically efficient t-norms and t-conorms.

Other uncertainty-related formulas can also be similarly explained [11].

What we do in this paper. In this paper, on two detailed examples, we show that not only the basic formulas, but many other aspects of uncertainty can be explained in terms of symmetries: heuristic and semi-heuristic approaches can be justified by appropriate natural symmetries, and symmetries can help in designing optimal algorithms.

\section{Symmetries Help in Explaining Existing Algorithms: Case Study}

Practical need for uncertainty propagation. In many practical situations, we are interested in the value of a quantity $y$ which is difficult or even impossible to measure directly. To estimate this difficult-to-measure quantity $y$, we measure or estimate related easier-to-measure quantities $x_{1}, \ldots, x_{n}$ which are related to the desired quantity $y$ by a known relation $y=f\left(x_{1}, \ldots, x_{n}\right)$. Then, we apply the relation $f$ to the estimates $\widetilde{x}_{1}, \ldots, \widetilde{x}_{n}$ for $x_{i}$ and produce an estimate $\widetilde{y}=f\left(\widetilde{x}_{1}, \ldots, \widetilde{x}_{n}\right)$ for the desired quantity $y$.

In the simplest cases, the relation $f\left(x_{1}, \ldots, x_{n}\right)$ may be an explicit expression: e.g., if we know the current $x_{1}$ and the resistance $x_{2}$, then we can measure the voltage $y$ by using Ohm's law $y=x_{1} \cdot x_{2}$. In many practical situations, the relation between $x_{i}$ and $y$ is much more complicated: the corresponding algorithm $f\left(x_{1}, \ldots, x_{n}\right)$ is not an explicit expression, but a complex algorithm for solving an appropriate non-linear equation (or system of equations).

Estimates are never absolutely accurate:

- measurements are never absolutely precise, and

- expert estimates can only provide approximate values of the directly measured quantities $x_{1}, \ldots, x_{n}$.

In both cases, the resulting estimates $\widetilde{x}_{i}$ are, in general, different from the actual (unknown) values $x_{i}$. Due to these estimation errors $\Delta x_{i} \stackrel{\text { def }}{=} \widetilde{x}_{i}-x_{i}$, even if the relation $f\left(x_{1}, \ldots, x_{n}\right)$ is exact, the estimate $\widetilde{y}=f\left(\widetilde{x}_{1}, \ldots, \widetilde{x}_{n}\right)$ is different from the actual value $y=f\left(x_{1}, \ldots, x_{n}\right): \Delta y \stackrel{\text { def }}{=} \widetilde{y}-y \neq 0$. 
(In many situations, when the relation $f\left(x_{1}, \ldots, x_{n}\right)$ is only known approximately, there is an additional source of the approximation error in $y$ caused by the uncertainty in knowing this relation.)

It is therefore desirable to find out how the uncertainty $\Delta x_{i}$ in estimating $x_{i}$ affects the uncertainty $\Delta y$ in the desired quantity, i.e., how the uncertainties $\Delta x_{i}$ propagate via the algorithm $f\left(x_{1}, \ldots, x_{n}\right)$.

Propagation of probabilistic uncertainty. Often, we know the probabilities of different values of $\Delta x_{i}$. For example, in many cases, we know that the approximation errors $\Delta x_{i}$ are independent normally distributed with zero mean and known standard deviations $\sigma_{i}$; see, e.g., [14].

In this case, we can use known statistical techniques to estimate the resulting uncertainty $\Delta y$ in $y$. For example, since we know the probability distributions, we can simulate them in the computer, i.e., use the Monte-Carlo simulation techniques to get a sample population $\Delta y^{(1)}, \ldots, \Delta y^{(N)}$ of the corresponding errors $\Delta y$. Based on this sample, we can then estimate the desired statistical characteristics of the desired approximation error $\Delta y$.

Propagation of interval uncertainty. In many other practical situations, we do not know these probabilities, we only know the upper bounds $\Delta_{i}$ on the (absolute values of) the corresponding measurement errors $\Delta x_{i}:\left|\Delta x_{i}\right| \leq \Delta$.

In this case, based on the known approximation $\widetilde{x}_{i}$, we can conclude that the actual (unknown) value of $i$-th auxiliary quantity $x_{i}$ can take any value from the interval

$$
\mathbf{x}_{i}=\left[\widetilde{x}_{i}-\Delta_{i}, \widetilde{x}_{i}+\Delta_{i}\right]
$$

To find the resulting uncertainty in $y$, we must therefore find the range $\mathbf{y}=[y, \bar{y}]$ of possible values of $y$ when $x_{i} \in \mathbf{x}_{i}$ :

$$
\mathbf{y}=f\left(\mathbf{x}_{1}, \ldots, \mathbf{x}_{n}\right) \stackrel{\text { def }}{=}\left\{f\left(x_{1}, \ldots, x_{n}\right) \mid x_{1} \in \mathbf{x}_{1}, \ldots, x_{n} \in \mathbf{x}_{n}\right\} .
$$

Computations of this range under interval uncertainty is called interval computations; see, e.g., [6].

Comment. It is well known that processing fuzzy uncertainty can be reduced to processing interval uncertainty: namely, the $\alpha$-cut $\mathbf{y}(\alpha)$ for $y=f\left(x_{1}, \ldots, x_{n}\right)$ is equal to the range $f\left(\mathbf{x}_{1}(\alpha), \ldots, \mathbf{x}_{n}(\alpha)\right)$; see, e.g., [13].

Linearization. In many practical situations, the approximation errors $\Delta x_{i}=\widetilde{x}_{i}-x_{i}$ are small. In such situations, we can expand the expression for $\Delta y=\widetilde{y}-y$ in Taylor series in $\Delta x_{i}$ and keep only the linear terms in this expansion. In this case, we get $\Delta y=c_{1} \cdot \Delta x_{1}+\ldots+c_{n} \cdot \Delta x_{n}$, where $c_{i} \stackrel{\text { def }}{=} \frac{\partial f}{\partial x_{i}}\left(\widetilde{x}_{1}, \ldots, \widetilde{x}_{n}\right)$. So if $\left|\Delta x_{i}\right| \leq \Delta$, then $|\Delta y| \leq \Delta$, where

$$
\Delta=\left|c_{1}\right| \cdot \Delta_{1}+\ldots+\left|c_{n}\right| \cdot \Delta_{n} .
$$

For complex $f$, we can find $c_{i}$ by numerical differentiation. To estimate $n$ partial derivatives, we need $n$ calls to $f$. For large $n$ and complex $f$, this is too timeconsuming. 
Cauchy deviate method. For large $n$, we can further reduce the number of calls to $f$ if we use a special technique of Cauchy-based Monte-Carlo simulations, which enables us to use a fixed number of calls to $f(\approx 200)$ for all possible values $n$; see, e.g., [7]. This method uses Cauchy distribution - i.e., probability distributions with the probability density $\rho(z)=\frac{\Delta}{\pi \cdot\left(z^{2}+\Delta^{2}\right)}$; the value $\Delta$ is called the (scale) parameter of this distribution.

Cauchy distribution has the following property that we will use: if $z_{1}, \ldots, z_{n}$ are independent random variables, and each of $z_{i}$ is distributed according to the Cauchy law with parameter $\Delta_{i}$, then their linear combination

$$
z=c_{1} \cdot z_{1}+\ldots+c_{n} \cdot z_{n}
$$

is also distributed according to a Cauchy law, with a scale parameter $\Delta=\left|c_{1}\right| \cdot \Delta_{1}+$ $\ldots+\left|c_{n}\right| \cdot \Delta_{n}$.

Therefore, if we take random variables $\delta_{i}$ which are Cauchy distributed with parameters $\Delta_{i}$, then the value

$$
\begin{gathered}
\delta \stackrel{\text { def }}{=} f\left(\widetilde{x}_{1}, \ldots, \widetilde{x}_{n}\right)-f\left(\widetilde{x}_{1}-\delta_{1}, \ldots, \widetilde{x}_{n}-\delta_{n}\right)= \\
c_{1} \cdot \delta_{1}+\ldots+c_{n} \cdot \delta_{n}
\end{gathered}
$$

is Cauchy distributed with the desired parameter $\Delta=\sum_{i=1}^{n}\left|c_{i}\right| \cdot \Delta_{i}$.

Need for intuitive explanation. The Cauchy deviate method is one of the most efficient techniques for processing interval and fuzzy data. However, this method has a serious drawback: while the corresponding technique is mathematically valid, it is somewhat counterintuitive - we want to analyze errors which are located instead a given interval $[-\Delta, \Delta]$, but this analysis use Cauchy simulated errors which are located, with a high probability, outside this interval.

It is therefore desirable to come up with an intuitive explanation for this technique.

Our main idea: use neurons. Our explanation comes from the idea promoted by Paul Werbos, the author of the backpropagation algorithm for training neural networks. Traditionally, neural networks are used to simulate a deterministic dependence; Paul Werbos suggested that the same neural networks can be used to describe stochastic dependencies as well - if as one of the inputs, we take a standard random number $r$ uniformly distributed on the interval [0,1]; see, e.g., [18] and references therein.

In view of this idea, as a natural probability distribution, we can take the result of applying a neural network to this random number. The simplest case is when we have a single neuron. In this case, we apply the activation (input-output) function $f(y)$ corresponding to this neuron to the random number $r$.

Using appropriate symmetries. In [11], we described all activation functions $f(x)$ which are optimal with respect to reasonable symmetry-based criteria. It turns out 
that all such functions have the form $a+b \cdot f_{0}(K \cdot y+l)$, where $f_{0}(y)$ is either a linear function, or a fractional-linear function, or $f_{0}(y)=\exp (y)$, or the logistic (sigmoid) function $f_{0}(y)=1 /(1+\exp (-y))$, or $f_{0}(y)=\tan (y)$. The logistic function is indeed the most popular activation function for actual neural networks, but others are also used. For our purpose, we will use the tangent function: its application of the tangent function to the standard random number $r$ indeed leads to the desired Cauchy distribution.

\section{Symmetries Help in Designing Optimal Algorithms: Case Study}

Symmetries not only help to find the appropriate representations of uncertainty and appropriate formulas for processing uncertainty: symmetries also help to select the optimal algorithms for implementing the corresponding mathematical formulas.

Fixed points: a practical problem. In many real-life situations, we have dynamical situations which eventually reach an equilibrium.

For example, in economics, when a situation changes, prices start changing (often fluctuating) until they reach an equilibrium between supply and demand.

In transportation, when a new road is built, some traffic moves to this road to avoid congestion on the other roads; this causes congestion on the new road, which, in its turn, leads drivers to go back to their previous routes, etc. [15].

To describe the problem of finding the equilibrium state(s), we must first be able to describe all possible states. In this paper, we assume that we already have such a description, i.e., that we know the set $X$ of all possible states.

We must also be able to describe the fact that many states $x \in X$ are not equilibrium states. For example, if the price of some commodity (like oil) is set up too high, it will become profitable to explore difficult-to-extract oil fields; as a new result, the supply of oil will increase, and the prices will drop.

Similarly, as we have mentioned in the main text, if too many cars move to a new road, this road may become even more congested than the old roads initially were, and so the traffic situation will actually decrease - prompting people to abandon this new road.

To describe this instability, we must be able to describe how, due to this instability, the original state $x$ gets transformed in the next moment of time. In other words, we assume that for every state $x \in X$, we know the corresponding state $f(x)$ at the next moment of time.

For non-equilibrium states $x$, the change is inevitable, so we have $f(x) \neq x$. The equilibrium state $x$ is the state which does not change, i.e., for which $f(x)=x$. Thus, we arrive at the following problem: We are given a set $X$ and a function $f: X \rightarrow X$; we need to find an element $x$ for which $f(x)=x$.

In mathematical terms, an element $x$ for which $f(x)=x$ is called a fixed point of the mapping $f$. So, there is a practical need to find fixed points. 
The problem of computing fixed points. Since there is a practical need to compute the fixed points, let us give a brief description of the existing algorithms for computing these fixed points; see, e.g., [1].

Straightforward algorithm: Picard iterations. At first glance, the situation seems very simple and straightforward. We know that if we start with a state $x$ at some moment of time, then in the next moment of time, we will get a state $f(x)$. We also know that eventually, we will get an equilibrium. So, a natural thing to do is to simulate how the actual equilibrium will be reached.

In other words, we start with an arbitrary (reasonable) state $x_{0}$. After we know the state $x_{k}$ at the moment $k$, we predict the state $x_{k+1}$ at the next moment of time as $x_{k+1}=f\left(x_{k}\right)$. This algorithm is called Picard iterations after a mathematician who started efficiently using it in the 19 century.

If the equilibrium is eventually achieved, i.e., if in real life the process converges to an equilibrium point $x$, then Picard's iterations are guaranteed to converge. Their convergence may be somewhat slow - since they simulate all the fluctuations of the actual convergence - but eventually, we get convergence.

Situations when Picard's iterations do not converge: economics. In some important practical situations, Picard iterations do not converge.

The main reason is that in practice, we can have panicky fluctuations which prevent convergence. Of course, one expects fluctuations. For example, if the price of oil is high, then it will become profitable for companies to explore and exploit new oil fields. As a result, the supply of oil will drastically increase, and the price of oil will go down. Since this is all done in a unplanned way, with different companies making very rough predictions, it is highly probable that the resulting oil supply will exceed the demand. As a result, prices will go down, oil production in difficult-toproduce oil areas will become unprofitable, supply will go down, etc.

Such fluctuations have happened in economics in the past, and sometimes, not only they did not lead to an equilibrium, they actually led to deep economic crises.

How can we handle these situation: a natural practical solution. If the natural Picard iterations do not converge, this means that in practice, there is too much of a fluctuation. When at some moment $k$, the state $x_{k}$ is not an equilibrium, then at the next moment of time, we have a state $x_{k+1}=f\left(x_{k}\right) \neq x_{k}$. However, this new state $x_{k+1}$ is an not necessarily closer to the equilibrium: it "over-compensates" by going too far to the other side of the desired equilibrium.

For example, we started with a price $x_{k}$ which was too high. At the next moment of time, instead of having a price which is closer to the equilibrium, we may get a new price $x_{k+1}$ which is too low - and may even be further away from the equilibrium than the previous price.

In practical situations, such things do happen. In this case, to avoid such weird fluctuations and to guarantee that we eventually converge to the equilibrium point, a natural thing is to "dampen" these fluctuations: we know that a transition from $x_{k}$ to $x_{k+1}$ has gone too far, so we should only go "halfway" (or even smaller piece of the way) towards $x_{k+1}$. 
How can we describe it in natural terms? In many practical situations, there is a reasonable linear structure on the set $X$ on all the states, i.e., $X$ is a linear space. In this case, going from $x_{k}$ to $f\left(x_{k}\right)$ means adding, to the original state $x_{k}$, a displacement $f\left(x_{k}\right)-x_{k}$. Going halfway would then mean that we are only adding a half of this displacement, i.e., that we go from $x_{k}$ to $x_{k+1}=x_{k}+\frac{1}{2} \cdot\left(f\left(x_{k}\right)-x_{k}\right)$, i.e., to

$$
x_{k+1}=\frac{1}{2} \cdot x_{k}+\frac{1}{2} \cdot f\left(x_{k}\right) \text {. }
$$

The corresponding iteration process is called Krasnoselskii iterations. In general, we can use a different portions $\alpha \neq 1 / 2$, and we can also use different portions $\alpha_{k}$ on different moments of time. In general, we thus go from $x_{k}$ to $x_{k+1}=x_{k}+\alpha_{k}$. $\left(f\left(x_{k}\right)-x_{k}\right)$, i.e., to

$$
x_{k+1}=\left(1-\alpha_{k}\right) \cdot x_{k}+\alpha_{k} \cdot f\left(x_{k}\right) \text {. }
$$

These iterations are called Krasnoselski-Mann iterations.

Practical problem: the rate of convergence drastically depends on $\alpha_{i}$. The above convergence results show that under certain conditions on the parameters $\alpha_{i}$, there is a convergence. From the viewpoint of guaranteeing this convergence, we can select any sequence $\alpha_{i}$ which satisfies these conditions. However, in practice, different choice of $\alpha_{i}$ often result in drastically different rate of convergence.

To illustrate this difference, let us consider the simplest situation when already Picard iterations $x_{n+1}=f\left(x_{n}\right)$ converge, and converge monotonically. Then, in principle, we can have the same convergence if instead we use Krasnoselski-Mann iterations with $\alpha_{n}=0.01$. Crudely speaking, this means that we replace each original step $x_{n} \rightarrow x_{n+1}=f\left(x_{n}\right)$, which bring $x_{n}$ directly into $x_{n+1}$, by a hundred new smaller steps. Thus, while we still have convergence, we will need 100 times more iterations than before - and thus, we require a hundred times more computation time.

Since different values $\alpha_{i}$ lead to different rates of convergence, ranging from reasonably efficient to very inefficient, it is important to make sure that we select optimal values of the parameters $\alpha_{i}$, values which guarantee the fastest convergence.

Idea: from the discrete iterations to the continuous dynamical system. In this section, we will describe the values $\alpha_{i}$ which are optimal in some reasonable sense. To describe this sense, let us go back to our description of the dynamical situation. In the above text, we considered observations made at discrete moments of time; this is why we talked about current moment of time, next moment of time, etc. In precise terms, we considered moments $t_{0}, t_{1}=t_{0}+\Delta t, t_{2}=t_{0}+2 \Delta t$, etc.

In principle, the selection of $\Delta t$ is rather arbitrary. For example, in terms of prices, we can consider weekly prices (for which $\Delta t$ is one week), monthly prices, yearly prices, etc. Similarly, for transportation, we can consider daily, hourly, etc. descriptions. The above discrete-time description is, in effect, a discrete approximation to an actual continuous-time system.

Similarly, Krasnoselski-Mann iterations $x_{k+1}-x_{k}=\alpha_{k} \cdot\left(f\left(x_{k}\right)-x_{k}\right)$ can be viewed as a discrete-time approximations to a continuous dynamical system which 
leads to the desired equilibrium. Specifically, the difference $x_{k+1}-x_{k}$ is a natural discrete analogue of the derivative $\frac{d x}{d t}$, the values $\alpha_{k}$ can be viewed as discretized values of an unknown function $\alpha(t)$, and so the corresponding continuous system takes the form

$$
\frac{d x}{d t}=\alpha(t) \cdot(f(x)-x)
$$

A discrete-time system is usually a good approximation to the corresponding continuous-time system. Thus, we can assume that, vice versa, the above continuous system is a good approximation for Krasnoselski-Mann iterations.

In view of this fact, in the following text, we will look for an appropriate (optimal) continuous-time system (7).

Scale invariance: natural requirement on a continuous-time system. In deriving the continuous system (7) from the formula for Krasnoselski-Mann iterations, we assumed that the original time interval $\Delta t$ between the two consecutive iterations is 1. This means, in effect, that to measure time, we use a scale in which this interval $\Delta t$ is a unit interval.

As we have mentioned earlier, the choice of the time interval $\Delta t$ is rather arbitrary. If we make a different choice of this discretization time interval $\Delta t^{\prime} \neq \Delta t$, then we would get a similar dynamical system, but described in a different time scale, with a different time interval $\Delta t^{\prime}$ taken as a measuring unit. As a result of "de-discretizing" this new system, we would get a different continuous system of type (7) - a system which differs from the original one by a change in scale.

In the original scale, we identified the time interval $\Delta t$ with 1 . Thus, the time $t$ in the original scale means physical time $T=t \cdot \Delta t$. In the new scale, this same physical time corresponds to the time $t^{\prime}=\frac{T}{\Delta t^{\prime}}=t \cdot \frac{\Delta t}{\Delta t^{\prime}}$.

If we denote by $\lambda=\frac{\Delta t^{\prime}}{\Delta t}$ the ratio of the corresponding units, then we conclude that the time $t$ in the original scale corresponds to the time $t^{\prime}=t / \lambda$ in the new scale. Let us describe the system (7) in terms of this new time coordinate $t^{\prime}$. From the above formula, we conclude that $t=\lambda \cdot t^{\prime}$; substituting $t=\lambda \cdot t^{\prime}$ and $d t=\lambda \cdot d t^{\prime}$ into the formula (7), we conclude that $\frac{1}{\lambda} \cdot \frac{d x}{d t^{\prime}}=\alpha\left(\lambda \cdot t^{\prime}\right) \cdot(f(x)-x)$, i.e., that

$$
\frac{d x}{d t^{\prime}}=\left(\lambda \cdot \alpha\left(\lambda \cdot t^{\prime}\right)\right) \cdot(f(x)-x)
$$

It is reasonable to require that the optimal system of type (7) should not depend on what exactly time interval $\Delta t$ we used for discretization.

Conclusion: optimal Krasnoselski-Mann iterations correspond to $\alpha_{k}=c / k$. Since a change of the time interval corresponds to re-scaling, this means the system (7) must be scale-invariant, i.e., to be more precise, the system (8) must have exactly the same form as the system (7) but with $t^{\prime}$ instead of $t$, i.e., the form

$$
\frac{d x}{d t^{\prime}}=\alpha\left(t^{\prime}\right) \cdot(f(x)-x)
$$


By comparing the systems (8) and (9), we conclude that we must have $\lambda \cdot \alpha\left(\lambda \cdot t^{\prime}\right)=$ $a\left(t^{\prime}\right)$ for all $t^{\prime}$ and $\lambda$. In particular, if we take $\lambda=1 / t^{\prime}$, then we get $\alpha\left(t^{\prime}\right)=\frac{\alpha(1)}{t^{\prime}}$, i.e., $\alpha\left(t^{\prime}\right)=c / t^{\prime}$ for some constant $c(=\alpha(1))$.

With respect to the corresponding discretized system, this means that we take $\alpha_{k}=\alpha(k)=c / k$.

This selection works well. Our experiments on transportation problems confirmed that this procedure converges $[2,3]$.

The choice $a_{k}=1 / k$ have been successfully used in other applications as well; see, e.g., [17] and references therein.

\section{Other Economics-Related Examples: In Brief}

In economics, scale-invariance explains empirical formulas for economic fluctuations [9] and for risk analysis [12], and the use of Choquet integrals [10]. A combination of scale- and shift-invariance explains Hurwicz's empirical formula for decision making under interval uncertainty [5].

Nonlinear transformation groups explain heuristic formulas describing volatility and financial risk [12].

\section{Conclusion}

One of the main objectives of science is to predict future events, in particular, the results of different actions. Many such predictions are based on the notion of invariance: we already know how similar situations evolved, so we can conclude that the current situation will evolve in a similar way. As a result, the ideas based on invariance and symmetry are among the main tools of modern physics: these ideas provide a precise justification for empirically justified heuristic formulas, these ideas lead to efficient algorithms for solving physical problems.

In this paper, we show that similar invariance ideas can explain heuristic formulas and algorithms related to processing different types of uncertainty, and that these ideas lead to efficient algorithms for solving problems under uncertainty. The efficiency of invariance ideas is illustrated on two detailed examples; several other applications of these ideas are overviewed. The variety of these applications make us conjecture that the symmetry ideas can form a basis for integrated uncertainty management.

Acknowledgements This work was supported in part by the Faculty of Economics at Chiang Mai University, Thailand, by the National Science Foundation grants HRD-0734825 and DUE0926721, and by Grant 1 T36 GM078000-01 from the National Institutes of Health.

The authors are thankful to the anonymous referees for valuable suggestions. 


\section{References}

1. V. Berinde, Iterative approximation of fixed points (Editura Efemeride, Baia Mare, 2002)

2. R. Cheu, V. Kreinovich, et al., Strategies for Improving Travel Time Reliability, Texas Department of Transportation, Research Report 0-5453-R2, 2007

3. R. Cheu, V. Kreinovich, F. Modave, G. Xiang, and T. Magoc, "How to estimate, take into account, and improve travel time reliability in transportation networks", In: R.L. Muhanna and R.L. Mullen (eds.) Proc. Int'l Workshop on Reliable Engineering Computing REC'08, Savannah, Georgia, February 20-22, 2008, pp. 289-332

4. R. Feynman, R. Leighton, and M. Sands, The Feynman Lectures on Physics (Addison Wesley, Boston, Massachusetts, 2005)

5. V.N. Huynh, C. Hu, Y. Nakamori, and V. Kreinovich, "On Decision Making under Interval Uncertainty: A New Justification of Hurwicz Optimism-Pessimism Approach and Its Use in Group Decision Making", Proceedings of the 39th International Symposium on MultipleValued Logic ISMVL'2009, Naha, Okinawa, Japan, May 21-23, 2009, pp. 214-220.

6. L. Jaulin et al., Applied Interval Analysis (Springer-Verlag, London, 2001)

7. V. Kreinovich and S. Ferson, "A New Cauchy-Based Black-Box Technique for Uncertainty in Risk Analysis", Reliability Eng. \& Systems Safety, 2004, Vol. 85, No. 1-3, pp. 267-279.

8. V. Kreinovich and H.T. Nguyen, "Towards A Neural-Based Understanding of the Cauchy Deviate Method for Processing Interval and Fuzzy Uncertainty", Proc. IFSA'2009, Lisbon, Portugal, July 20-24, 2009, pp. 1264-1269.

9. T. Magoc and V. Kreinovich, "Empirical Formulas for Economic Fluctuations: Towards A New Justification", Proceedings of the 28th North American Fuzzy Information Processing Society Annual Conference NAFIPS'09, Cincinnati, Ohio, June 14-17, 2009.

10. F. Modave, M. Ceberio, and V. Kreinovich, "Choquet Integrals and OWA Criteria as a Natural (and Optimal) Next Step After Linear Aggregation: A New General Justification”, In: A. Gelbukh and E.F. Morales (eds.), Proceedings of the 7th Mexican International Conference on Artificial Intelligence MICAI'08, Mexico City, Mexico, October 27-31, 2008, Springer Lecture Notes on Artificial Intelligence, 2008, Vol. 5317, pp. 741-753.

11. H.T. Nguyen and V. Kreinovich, Applications of Continuous Mathematics to Computer Science (Kluwer, Dordrecht, 1997)

12. H.T. Nguyen, V. Kreinovich, and S. Sriboonchitta, "Stochastic Volatility Models and Financial Risk Measures: Towards New Justifications", Proceedings of the 2009 Singapore Economic Review Conference, Singapore, August 6-8, 2009.

13. H.T. Nguyen and E.A. Walker, A First Course in Fuzzy Logic (CRC Press, Boca Raton, Florida, 2006)

14. S. Rabinovich, Measurement Errors and Uncertainties: Theory and Practice (American Institute of Physics, New York, 2005)

15. Y. Sheffi, Urban Transportation Networks (Prentice Hall, Englewood Cliffs, NJ, 1985)

16. I.M. Singer and S. Sternberg, "Infinite groups of Lie and Cartan, Part 1", Journal d'Analyse Mathematique, 1965, Vol. XV, pp. 1-113.

17. Y. Su and X. Qin, "Strong convergence theorems for asymptotically nonexpansive mappings and asymptotically nonexpansive semigroups", Fixed Point Theory and Applications, 2006, Article ID 96215, pp. 1-11

18. P.J. Werbos, "Intelligence in the brain: A theory of how it works and how to build it", Neural Networks, 2009, Vol. 22, No. 3, pp. 200-212.

19. N. Wiener, Cybernetics, or Control and Communication in the animal and the machine (MIT Press, Cambridge, MA, 1962) 